\title{
VERIFICACIÓN DEL MODELO HARMONIE-AROME CONFIGURADO A MUY ALTA RESOLUCIÓN
}

\author{
Ruiz Pacheco, J. A., Padorno Prieto, E., Santos Atienza, I., Martínez Marco, I. \\ Agencia Estatal de Meteorología (AEMET), C/ Leonardo Prieto Castro, № 8
}

\section{Introducción}

Con el objetivo de evaluar la calidad de las predicciones obtenidas con el modelo de predicción numérica del tiempo HARMONIE-AROME a muy alta resolución $(1 \mathrm{~km})$, se han estudiado distintas zonas con el fin de examinar los resultados y compararlos frente a las salidas del modelo operativo a resolución $2,5 \mathrm{~km}$ y a los datos obtenidos por la red de estaciones meteorológicas automáticas (EMAs) de AEMET. Para ello, se ha empleado la herramienta MONITOR, desarrollada por el consorcio HIRLAM (High Resolution Limited Area Model).

Este estudio se ha realizado en el marco del proyecto SAMOA (Sistema de Apoyo Meteorológico y Oceanográfico para las Autoridades portuarias), que pretende dotar a los puertos de sistemas de consulta y explotación de la información oceanográfica y meteorológica personalizados. En particular, este estudio entra dentro del módulo de predicción meteorológica del proyecto.

Se han verificado distintas variables meteorológicas, en especial el viento a 10 metros sobre la superficie y la temperatura a 2 metros en ejecuciones del modelo en cuatro regio-

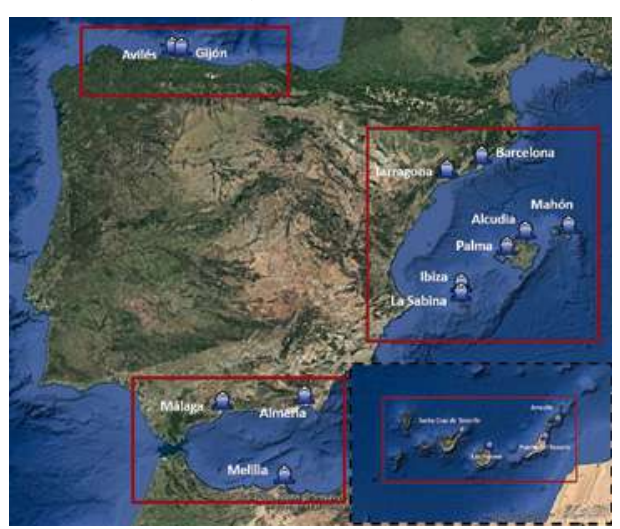
nes (Cantábrico, Alborán, Mediterráneo y Canarias) que engloban los puertos de interés para el proyecto, como se puede observar en la figura 1 . Se han realizado simulaciones con distintas escalas temporales: tanto a largo plazo, en estudios del comportamiento general durante meses completos como a corto plazo en situaciones de vientos intensos.

Fig. 1.- Regiones de estudio del modelo HARMONIE

\section{Descripción del modelo HARMONIE}

El modelo HARMONIE (Hirlam-Aladin Research in Mesoscale Operational NWP In Europe) es un modelo mesoescalar de predicción numérica espectral de área limitada que permite la convección, desarrollado por los consorcios HIRLAM y ALADIN (Aire Limitée Adaptation dynamique Développement InterNational). Su núcleo, ALADIN$\mathrm{NH}$, incorpora dinámica no hidrostática con un esquema semilagrangiano semiimplí- 
cito (Seity et al., 2011) en coordenadas horizontales cartesianas, mientras que la coordenada vertical es híbrida. En este estudio, se ejecuta la versión más reciente, v40h1.1 (Bengtsson et al., 2017).

Para altas resoluciones se emplea la configuración AROME puesto que permite resolver procesos subrejilla tales como la convección o la radiación solar. Para muy altas resoluciones, como en este caso, se debe adaptar la configuración actual de AROME a $2,5 \mathrm{~km}$ con el fin de evitar inestabilidad. Así, se han realizado una serie de cambios, como reducir el paso de tiempo a 30 segundos o emplear una topografía de mayor resolución. También se ha seleccionado un esquema numérico de resolución distinto al usual, el Predictor-Corrector; un esquema de difusión horizontal semilagrangiana (SLHD) en casi todas las variables, y el empleo del esquema HARATU, desarrollado por el grupo HIRLAM, que permite un cálculo de la turbulencia más realista (De Rooy et al., 2016; Cuxart et al., 2000).

En los experimentos realizados, se ha anidado HARMONIE al modelo IFS del ECMWF (European Centre for Medium-Range Weather Forecasts) en su ciclo 43r3, con una resolución aproximada de $9 \mathrm{~km}$.

\section{Herramienta MONITOR}

El modelo HARMONIE incluye un paquete de verificación denominado MONITOR. Este paquete permite el análisis estadístico de los datos de salida del modelo frente a los datos reales de observación y el cálculo de determinados valores estadísticos como pueden ser el sesgo o la desviación estándar, diagramas de dispersión e histogramas, así como el índice de Hansen-Kuiper u otras magnitudes significativas (Yang X., 2008).

La herramienta MONITOR realiza un control de calidad de los datos, eliminando las posibles anomalías estadísticas. A continuación, procesa los datos y elabora las salidas gráficas pertinentes mediante el paquete gráfico MAGICS del ECMWF o el programa de código libre gnuplot.

La principal ventaja de esta herramienta es su integración en el propio modelo: la salida del modelo puede ser utilizada sin necesidad de ser procesada. Además, puede usarse tanto de forma independiente como integrada en la ejecución. (HIRLAM documentation, 2014).

\section{Resultados de los experimentos}

Se ha utilizado la herramienta MONITOR para comparar los resultados del modelo HARMONIE-AROME a una resolución de $1 \mathrm{~km}$ frente a los resultados obtenidos por la salida operativa de AEMET, con una resolución de 2,5 km y a datos de la red de estaciones meteorológicas de AEMET. Así, hemos estudiado el comportamiento del viento a 10 metros, obteniendo tanto el sesgo y la desviación estándar, como el índice ETS (Equitable Threat Score) y diagramas de dispersión para los meses de marzo y octubre de 2017. También hemos estudiado la temperatura a 2 metros, obteniendo únicamente el sesgo y la desviación estándar para dichos meses, y un caso particular de vientos intensos producido durante enero de 2018. 


\subsection{Viento a 10 metros}

En primer lugar, veremos la verificación del viento a 10 metros durante los meses de marzo (izquierda) y octubre (derecha) de 2017 en las cuatro regiones anteriormente mencionadas. Para las siguientes gráficas, la serie roja hace referencia a los experimentos con resolución a $1 \mathrm{~km}$ y la serie verde a los de resolución a 2,5 km, mientras que las series con cuadrado (-ם) hacen referencia al sesgo y las series con asterisco (-*-) a la desviación estándar.
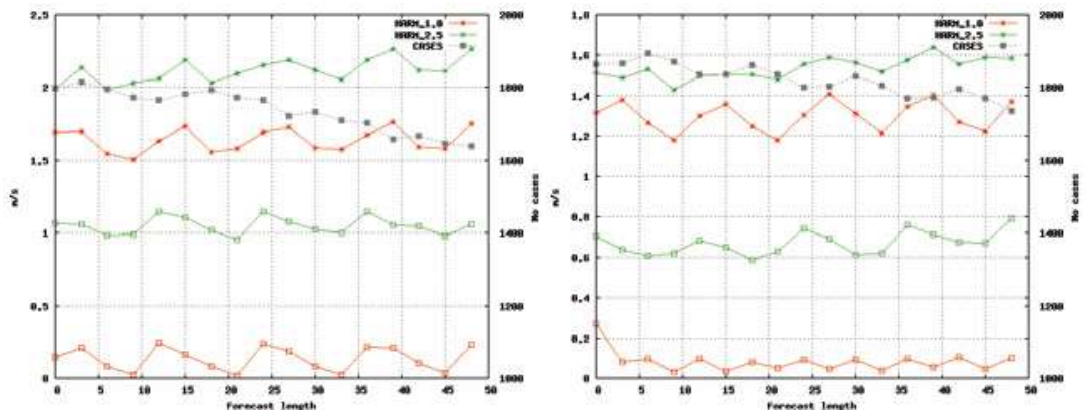

Fig. 2.- Sesgo y desviación estándar del viento a 10 metros en la zona del Cantábrico durante los meses de marzo de 2017 (izquierda) y octubre de 2017 (derecha)

En el caso del Cantábrico, podemos observar en la figura 2 que tanto el sesgo como la desviación estándar del experimento de $1 \mathrm{~km}$ son significativamente menores que los del experimento de $2.5 \mathrm{~km}$, tanto más en el caso del sesgo que en el de la desviación estándar, lo cual nos indica que los resultados para la serie de $1 \mathrm{~km}$ son mejores. Si comparamos los resultados del mes de marzo (izquierda) con el de octubre (derecha), observamos que ambas series, tanto sesgo como desviación estándar, son ligeramente mejores en el caso de octubre, mientras que la mejora no se ve significativamente afectada por la variación estacional. En cualquier caso, el sesgo es siempre positivo para ambos experimentos, debido a la tendencia del modelo a sobreestimar el viento. Dicha tendencia se ve drásticamente reducida en el experimento de $1 \mathrm{~km}$.

En cuanto a la zona de Alborán, vemos en la figura 3 que de nuevo el experimento de $1 \mathrm{~km}$ presenta mejoras frente al experimento de $2,5 \mathrm{~km}$, pero no son tan marcadas como en la región del Cantábrico, sino que son mucho menores. De nuevo, los resultados del mes de octubre son mejores en ambos experimentos, lo cual se puede achacar a una situación de estabilidad durante el mes de octubre que causó una mejor aproximación de los modelos a la realidad. También se confirma la tendencia a la sobreestimación del viento del modelo.

En el caso del Mediterráneo, se puede ver en la figura 4 un comportamiento mucho más similar al del Cantábrico, con una mejora en el experimento de $1 \mathrm{~km}$ bastante significativa respecto al de $2,5 \mathrm{~km}$, tanto más en el sesgo que en la desviación estándar.

En cuanto a la zona de Canarias, podemos observar en la figura 5 la misma tendencia que en las otras zonas. En particular, cabe notar el sesgo casi nulo que se produce durante el mes de octubre en el experimento de $1 \mathrm{~km}$, lo que nos indica que no existe una ten- 
dencia clara, puesto que los errores en ambos sentidos, menores que en el experimento de 2,5 km como indica la menor desviación estándar, tienden a compensarse entre sí.
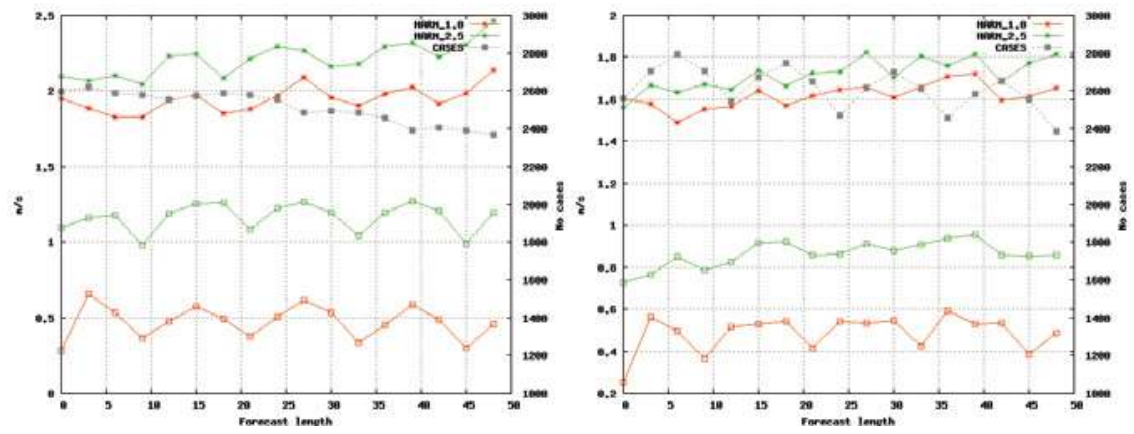

Fig. 3.- Sesgo y desviación estándar del viento a 10 metros en la zona de Alborán durante los meses de marzo de 2017 (izquierda) y octubre de 2017 (derecha)
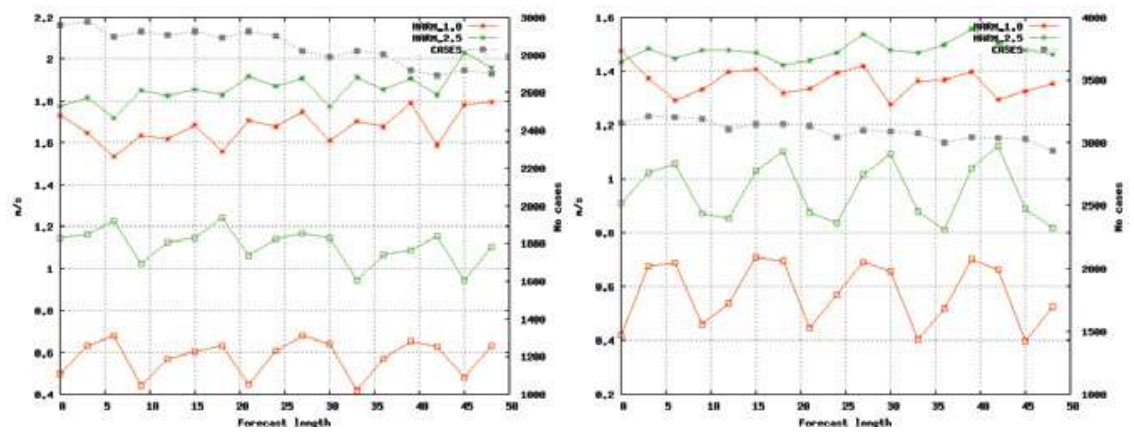

Fig. 4.- Sesgo y desviación estándar del viento a 10 metros en la zona del Mediterráneo durante los meses de marzo de 2017 (izquierda) y octubre de 2017 (derecha)
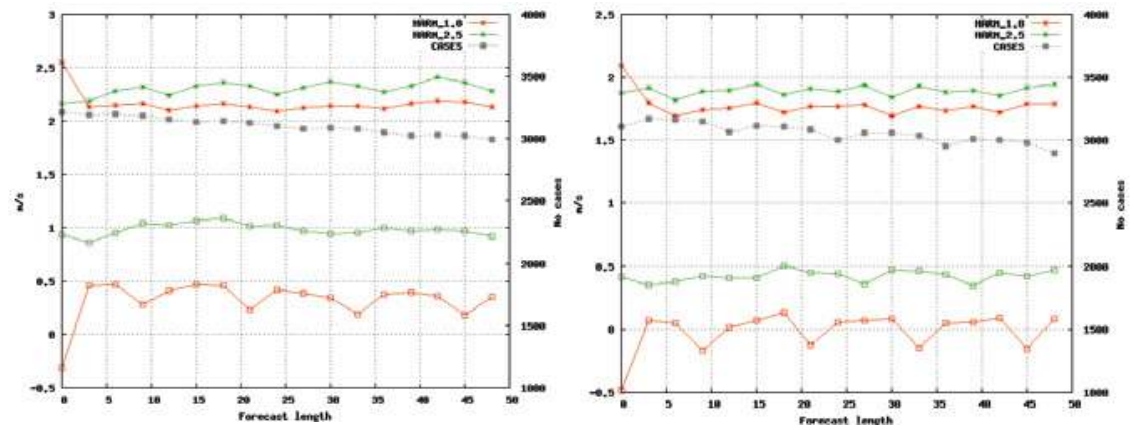

Fig. 5.- Sesgo y desviación estándar del viento a 10 metros en la zona de Canarias durante los meses de marzo de 2017 (izquierda) y octubre de 2017 (derecha) 


\subsection{Equitable Threat Score de viento a 10 metros}

Estudiaremos ahora los diagramas del índice ETS, que permiten la comparación de la tasa de aciertos frente a los fallos de ambos experimentos según categorías de viento. Este índice es especialmente apropiado puesto que elimina los resultados aleatorios, es decir, aquellos que se producen únicamente por azar (en climas húmedos, por ejemplo, se obtienen más resultados de precipitación que en climas secos, con el consiguiente mayor acierto en casos de precipitación que no tiene que ver con la capacidad del modelo, sino con las circunstancias del clima), con lo que podemos comparar mejor ambos experimentos. De nuevo, utilizamos la serie roja para los experimentos de $1 \mathrm{~km}$ y la serie verde para los de $2,5 \mathrm{~km}$, representando los meses de marzo a izquierda y octubre a derecha.
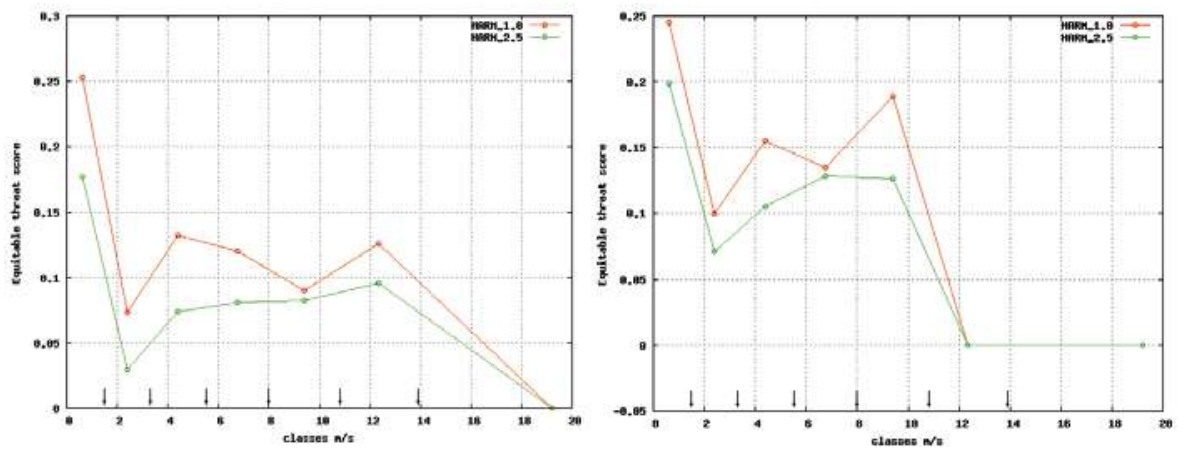

Fig. 6.- ETS para el viento a 10 metros de la zona del Cantábrico durante los meses de marzo de 2017 (izquierda) y octubre de 2017 (derecha)

Vemos en la figura 6 que en la zona del Cantábrico, el índice ETS del experimento de $1 \mathrm{~km}$ es superior al ETS de 2,5 km para todas las categorías de viento, especialmente en vientos débiles y moderados, lo cual indica una mayor tasa de aciertos del experimento de $1 \mathrm{~km}$.

En cuanto a la zona de Alborán podemos observar en la figura 7 que se obtienen unos resultados un poco más confusos. Si bien en el mes de marzo hay una notable diferencia entre los experimentos con una mejor estimación del viento por parte de las simulaciones de $1 \mathrm{~km}$, para el mes de octubre tenemos que dicha diferencia es mucho menor, llegando a existir categorías de vientos donde el modelo de $2,5 \mathrm{~km}$ representa mejor los resultados que el experimento de $1 \mathrm{~km}$.

En las zonas del Mediterráneo (figura 8) y Canarias (figura 9), se observa un comportamiento análogo al de las regiones anteriores, con los resultados del experimento de $1 \mathrm{~km}$ generalmente mejores que los de $2,5 \mathrm{~km}$, sobre todo en vientos débiles y moderados durante el mes de marzo, mientras que el experimento de $2,5 \mathrm{~km}$ puede presentar una ligera mejora en determinados casos puntuales. 

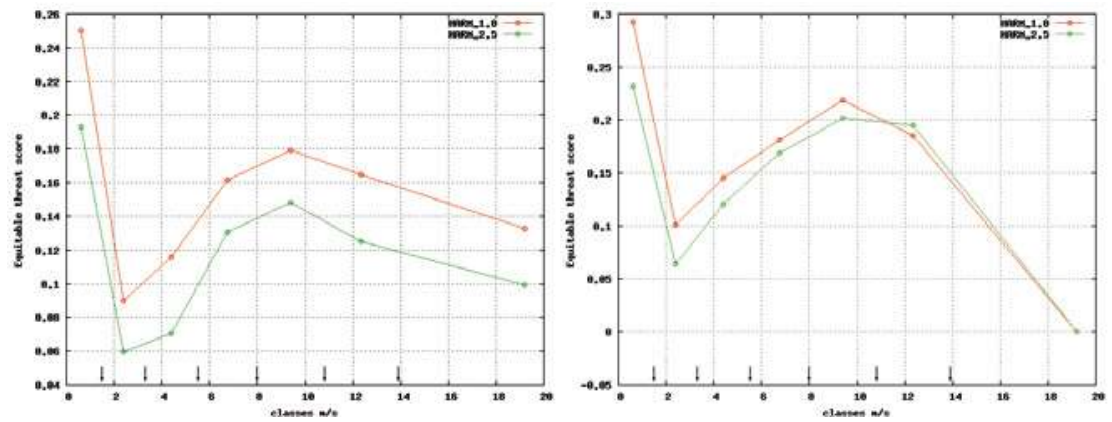

Fig. 7.- ETS para el viento a 10 metros de la zona de Alborán durante los meses de marzo de 2017 (izquierda) y octubre de 2017 (derecha)
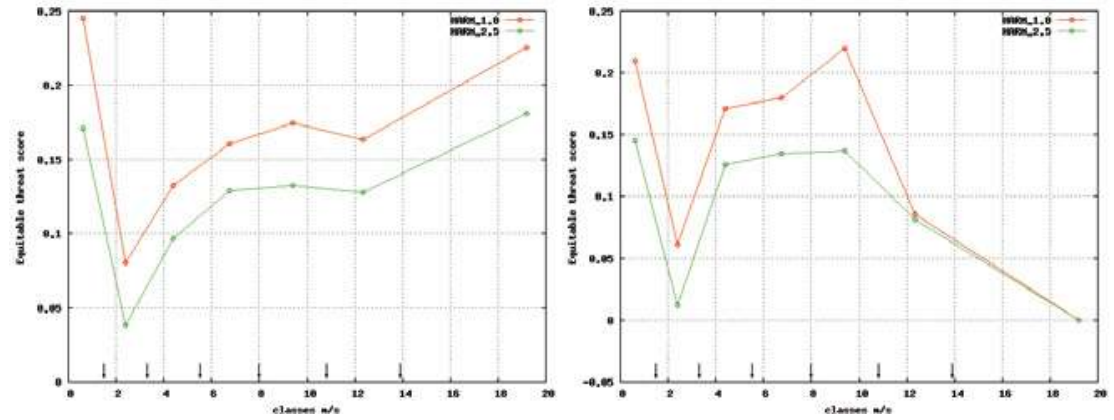

Fig. 8. ETS para el viento a 10 metros de la zona del Mediterráneo durante los meses de marzo de 2017 (izquierda) y octubre de 2017 (derecha)
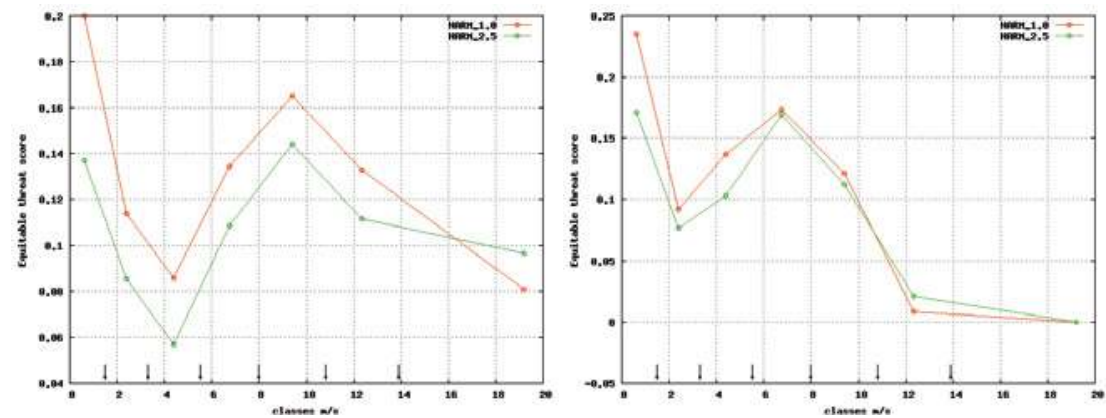

Fig. 9.- ETS para el viento a 10 metros de la zona de Canarias durante los meses de marzo de 2017 (izquierda) y octubre de 2017 (derecha) 


\subsection{Dispersión de viento a 10 metros}

A continuación se muestran los diagramas de dispersión para el viento a 10 metros en las cuatro regiones durante el mes de octubre de 2017. Los diagramas de la izquierda representan los datos del experimento de $1 \mathrm{~km}$ en el eje de ordenadas frente a las observaciones en el eje de abscisas, mientras que los de la derecha representan los datos de $2,5 \mathrm{~km}$.
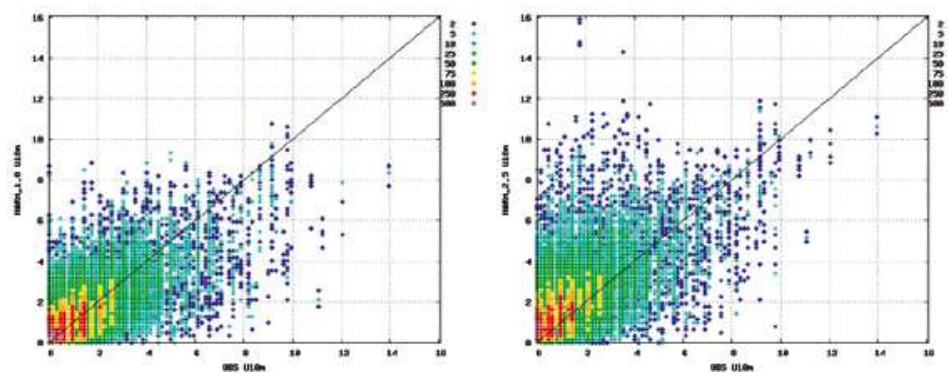

Fig. 10.- Diagrama de dispersión del viento a 10 metros de la zona del Cantábrico durante el mes de octubre de 2017 para los experimentos de resolución 1 km (izquierda) y 2,5 km (derecha)
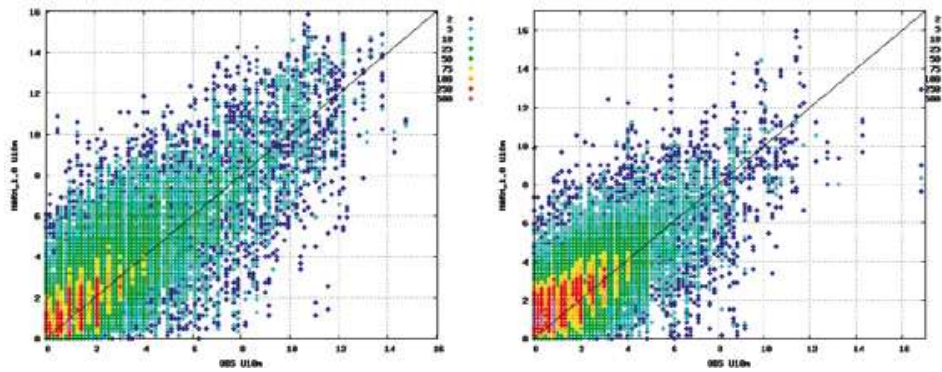

Fig. 11.- Diagrama de dispersión del viento a $\mathbf{1 0}$ metros de la zona de Alborán durante el mes de octubre de 2017 para los experimentos de resolución 1 km (izquierda) y 2,5 km (derecha)
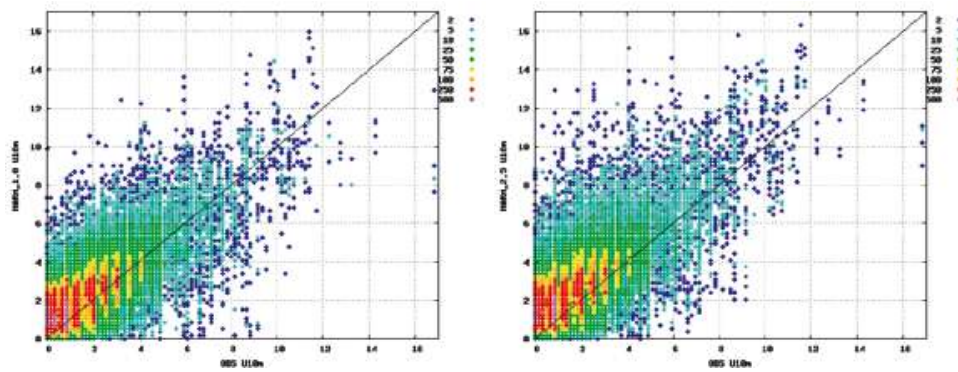

Fig. 12.- Diagrama de dispersión del viento a 10 metros de la zona del Mediterráneo durante el mes de octubre de 2017 para los experimentos de resolución $1 \mathrm{~km}$ (izquierda) y 2,5 km (derecha) 

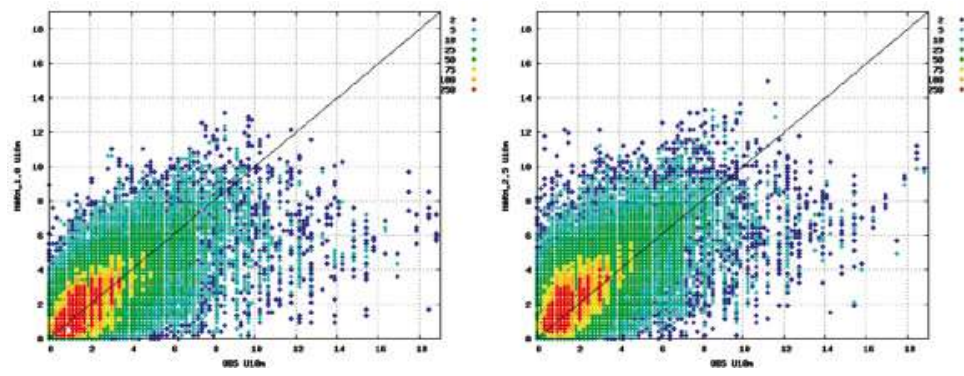

Fig. 13.-

Diagrama de dispersión del viento a 10 metros de la zona de Canarias durante el mes de octubre de 2017 para los experimentos de resolución $1 \mathrm{~km}$ (izquierda) y $2,5 \mathrm{~km}$ (derecha)

Podemos observar en la figura 10 que, para la zona del Cantábrico, el experimento de $1 \mathrm{~km}$ (a izquierda) presenta una serie de datos más compacta, lo cual concuerda con la menor desviación estándar del mismo, como vimos anteriormente. También vemos que los datos están más cerca de la diagonal que los del experimento de $2,5 \mathrm{~km}$, con lo cual su sesgo está más cercano al cero. Para el resto de regiones, es decir, Alborán (figura 11), Mediterráneo (figura 12) y Canarias (figura 13), vemos el mismo comportamiento, con pequeñas variaciones según la zona.

\subsection{Temperatura a 2 metros}

Para el estudio de la temperatura a 2 metros sobre la superficie en las zonas anteriormente mencionadas, seguiremos la misma pauta que en el caso del viento, comparando los meses de marzo (a izquierda) con octubre (a derecha) para los experimentos de $1 \mathrm{~km}$ (en rojo) y de 2,5 km (en verde). Utilizaremos las series de sesgo (-ם-) y de desviación estándar (-*-).
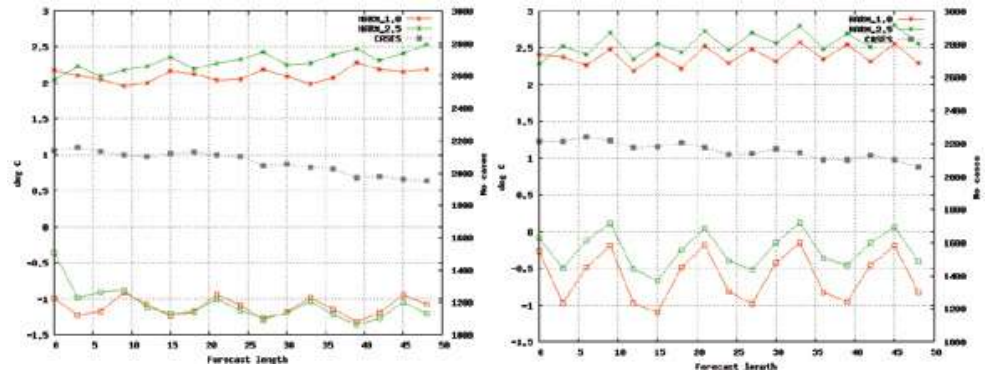

Fig. 14.- Sesgo y desviación estándar de la temperatura a 2 metros en la zona del Cantábrico durante los meses de marzo de 2017 (izquierda) y octubre de 2017 (derecha)

En la figura 14 podemos observar los resultados de la zona del Cantábrico, con una tendencia de la temperatura contraria al caso del viento: el modelo de $1 \mathrm{~km}$ mejora ligeramente el valor de la desviación estándar, aunque incrementa el sesgo negativo de esta magnitud ya existente en el experimento de 2,5 km. Este fenómeno es intrínseco al funcionamiento del modelo, donde una mejora en la representación del viento, reduciendo los valores antes obtenidos, lleva asociado un empeoramiento en la temperatura (de Rooy y de Vries, 2017). 
Para la zona de Alborán, podemos observar en la figura 15 el mismo comportamiento del Cantábrico pero de mayor magnitud. En este caso, durante el mes de marzo el sesgo presenta un empeoramiento notable, mientras que la desviación estándar apenas presenta mejoría. En el mes de octubre los cambios que se dan son casi inapreciables.

En cuanto al Mediterráneo, vemos en la figura 16 que si bien existe un empeoramiento en el sesgo, es menor que en la zona de Alborán, llegando al punto de que durante el mes de octubre el comportamiento del modelo de $1 \mathrm{~km}$ es casi idéntico al de 2,5 km.

Por último, en la zona de Canarias, se puede ver en la figura 17 que el comportamiento del modelo de $1 \mathrm{~km}$ sigue las mismas tendencias que en casos anteriores, pero de una magnitud menor, siendo similar al caso del Cantábrico, pero con un comportamiento distinto de la variación estacional.
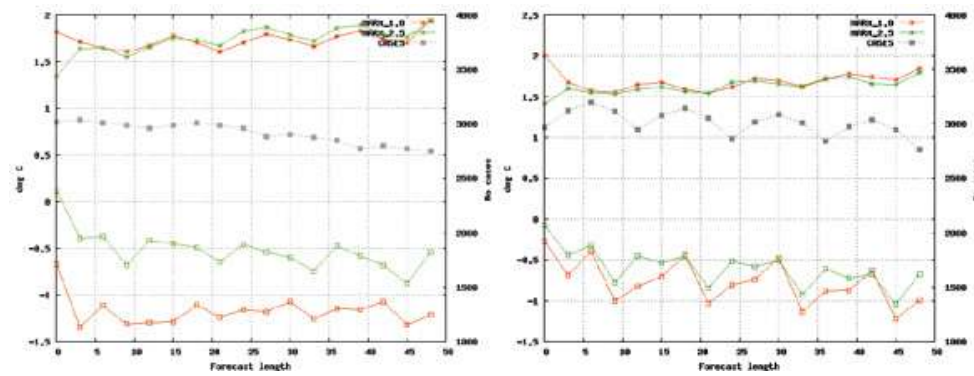

Fig. 15.- Sesgo y desviación estándar de la temperatura a 2 metros en la zona de Alborán durante los meses de marzo de 2017 (izquierda) y octubre de 2017 (derecha)
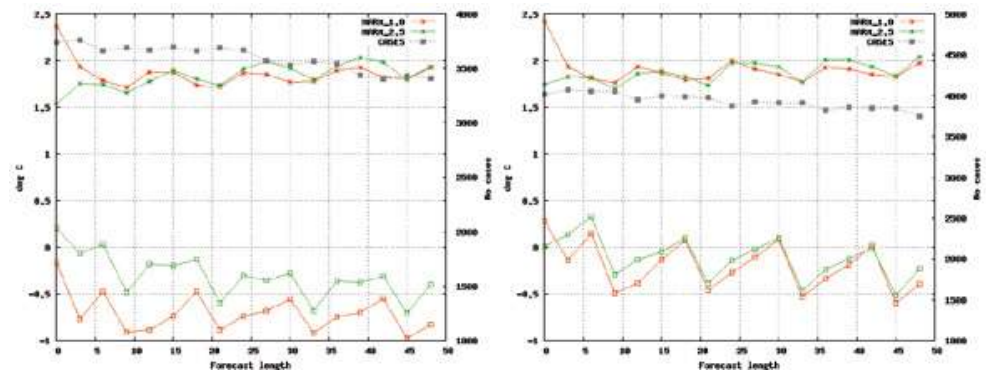

Fig. 16.- Sesgo y desviación estándar de la temperatura a 2 metros en la zona del Mediterráneo durante los meses de marzo de 2017 (izquierda) y octubre de 2017 (derecha)
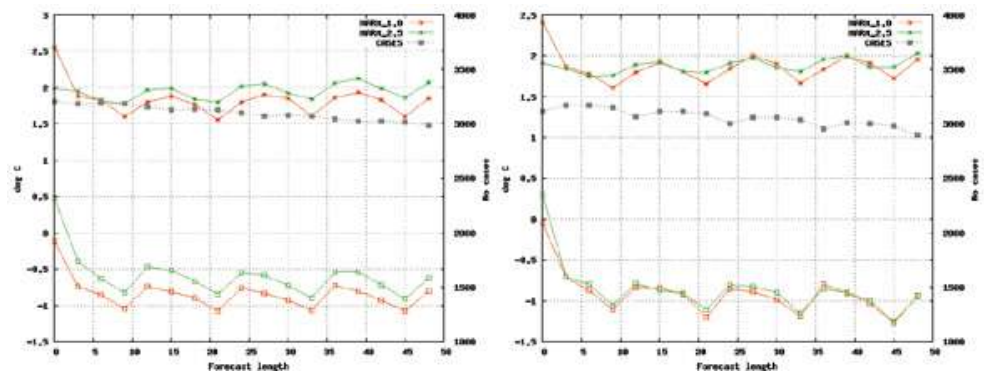

Fig. 17.- Sesgo y desviación estándar de la temperatura a 2 metros en la zona de Canarias durante los meses de marzo de 2017 (izquierda) y octubre de 2017 (derecha) 


\subsection{Caso de estudio de viento intenso}

Estudiaremos ahora los resultados de una semana con condiciones de viento intenso y fuerte convección, la semana del 8 al 15 de enero de 2018, en la zona del Cantábrico. En este tipo de situaciones, donde un modelo que resuelve la convección como puede ser HARMONIE se beneficia más de una mayor resolución que en el resto de casos, se confirma la obtención de mejores resultados.
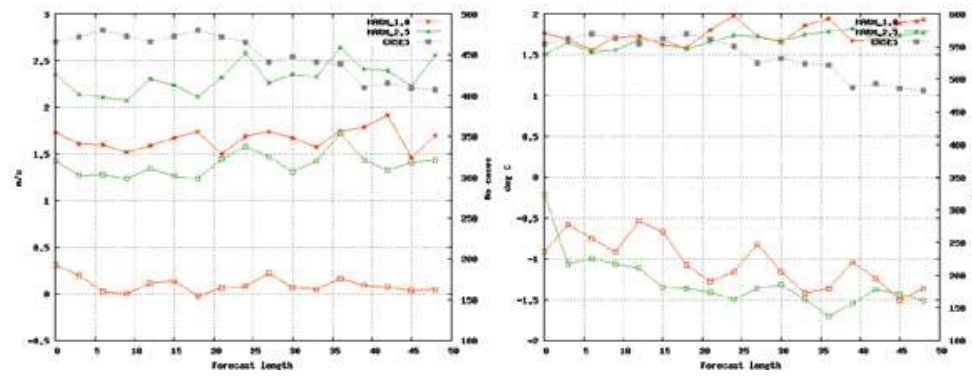

Fig. 18.- Sesgo y desviación estándar del viento a $\mathbf{1 0}$ metros (izquierda) y de la temperatura (derecha) en la zona del Cantábrico del 8 al 15 de enero de 2018

En la figura 18 podemos observar tanto el viento a 10 metros como la temperatura a 2 metros en la zona durante la semana de fuerte convección. Para el viento, tenemos que el experimento de $1 \mathrm{~km}$ presenta resultados excelentes, con un sesgo muy cercano al cero y una desviación estándar relativamente baja, mucho menores que los correspondientes al experimento de $2,5 \mathrm{~km}$. En cuanto a la temperatura, se observa que en este caso el experimento de $1 \mathrm{~km}$ registra una mejora del sesgo frente al de $2,5 \mathrm{~km}$, en lugar del empeoramiento anteriormente mostrado, corrigiendo de forma notable la mayor falla del modelo en estos casos.
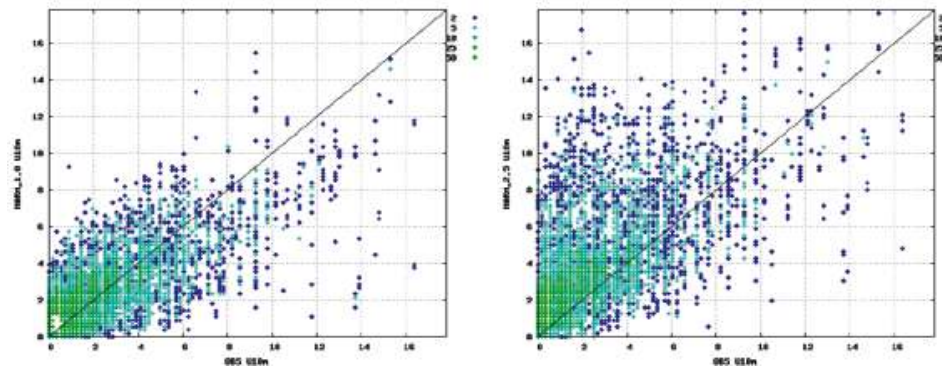

Fig. 19.- Diagrama de dispersión del viento a 10 metros de la zona del Cantábrico durante la semana del 8 al 15 de enero de 2018 para los experimentos de resolución $1 \mathrm{~km}$ (izquierda) y 2,5 km (derecha)

En la figura 19 se pueden observar los diagramas de dispersión del viento del experimento de $1 \mathrm{~km}$ (a izquierda) y de $2,5 \mathrm{~km}$ (a derecha) frente a datos de observaciones. De nuevo, el experimento de $1 \mathrm{~km}$ presenta unos resultados más próximos a la diagonal y más compactos, con lo cual confirmamos su mejor comportamiento frente al experimento de $2,5 \mathrm{~km}$, como pudimos determinar en la figura 18 . 


\section{Conclusiones y trabajo futuro}

El modelo HARMONIE-AROM con resolución de $1 \mathrm{~km}$ presenta una notable mejora en la predicción del viento a 10 metros, reduciendo la sobreestimación de la pasada operativa. Dicha mejora se ve condicionada por la región y, en menor medida, por la época del año, siendo el Cantábrico la zona donde el aumento de resolución aporta más beneficios y Alborán y Canarias la que presenta menores diferencias, mientras que la zona del Mediterráneo muestra resultados intermedios. En casos de vientos fuertes, dicha mejora es aún más significativa que en condiciones normales.

La temperatura presenta una relación inversa a la mejora del viento, pero de magnitud mucho más pequeña; este empeoramiento está condicionado a características del modelo. Otras variables como la presión o la humedad presentan mejoras casi imperceptibles.

En cuanto a líneas de trabajo futuro, aparte del mantenimiento y actualización del modelo, se está trabajando en la migración de las ejecuciones del modelo HARMONIE a $1 \mathrm{~km}$ de la plataforma del ECMWF al superordenador NIMBUS de AEMET. También se trabaja en el continuo estudio y mejora de la configuración a $1 \mathrm{~km}$, explorando nuevas estrategias, como puede ser el empleo de orografía subrejilla. Por último, se planea el uso de otros tipos de verificación para poder estudiar magnitudes que no se comportan bien bajo verificación punto a punto, como puede ser el caso de la precipitación.

\section{Referencias}

- Bengtsson L., U. Andrae, T. Aspelien, Y. Batrak, J. Calvo, W. C. de Rooy, E. Gleeson, B. HansenSass, M. Homleid, M. Hortal, K. Ivarsson, G. Lendering, S. Niemelä, K. P. Nielsen, J. Onvleed, L. Rontu, P. Samuelsson, D. Santos Muñoz, A. Subías, S. Tijm, V. Toll, X. Yang, M. Ødegaard Køltzowb, 2017: The HARMONIE-AROME Model Configuration in ALADIN-HIRLAM NWP System. Monthly Weather Review, 145, 1919-1935.

- Cuxart J., P. Bougeault, y J. L. Redelsperger, 2000: A turbulence scheme allowing for mesoscale and large-eddy simulations. Q. J. R. Meteorological Soc., 126, 1-100.

- de Rooy W. C., H. de Vries, G. Lenderink, P. Siebesma, B. van Ulft, E. Bazile, X. Yang, J. Barkmeijer, U. Andrae, T. Moene, et al, 2016: HARATU (update). (http://www.umr-cnrm.fr/aladin/IMG/pdf/ asm_wim_2016_nw.pdf)

- de Rooy W., H. de Vries, et al, 2017: HARMONIE verification and evaluation. HIRLAM Technical Report, No. 70.

- HIRLAM, 2016: HARMONIE documentation [online]. Disponible en (http://www.hirlam.org/ index.php/documentation/harmonie)

- Seity, Y., P. Brousseau, S. Malarden, G. Hello, P. Bernard, F. Bouttier, C. Lac, V. Masson, 2011: The AROME-France convective-scale operational model. Monthly Weather Review, 139, 976-991.

- Yang, X., 2008: Development of HIRLAM/HARMONIE monitoring system. HIRLAM Newslette, 54. 